\title{
A Nonlinear Three-Moment Model for Radiative Transfer in Spherical Symmetry
}

\author{
Ruo Li; Weiming Li; $†$ and Lingchao Zheng*
}

March 14, 2019

\begin{abstract}
We study the approximation of the radiative transfer equation with a relatively few moments in the spherically symmetric case. We propose a threemoment model based on choosing the beta distribution as the ansatz for the specific intensity. This ansatz enables our model to capture the anisotropy in the distribution function. The characteristic structure of the Riemann problem of the model is studied in detail. Numerical simulations demonstrate its validity in approximating the radiative transfer equation in the spherically symmetric case and its advantage in approximating highly anisotropic distribution functions in comparison to the $P_{n}$ method.
\end{abstract}

Keywords: Radiative transfer, spherical symmetry, moment method, anisotropic distrubution

\section{Introduction}

The radiative transfer equation (RTE) is essential for describing the propagation of radiation through absorbing and emitting medium [28, 26] and has applications in the fields of astrophysics [8], atmospheric physics [23] and optical imaging [18]. It is a high-dimensional integro-differential kinetic equation for the specific intensity

*HEDPS \& CAPT, LMAM \& School of Mathematical Sciences, Peking University, Beijing, China, email: rli@math.pku.edu.cn

${ }^{\dagger}$ Applied and Computational Mathematics Division, Beijing Computational Science Research Center, Beijing, China and Department of Mathematics, The Hong Kong University of Science \& Technology, Clear Water Bay, Kowloon, Hong Kong, China. email: liweiming@csrc . ac. cn

${ }^{\ddagger}$ School of Mathematical Sciences, Peking University, Beijing, China, email: lczheng@pku.edu.cn 
(also referred to as the distribution function in this paper) of the radiation. Due to its high-dimensionality, numerical methods to solve the RTE is a challenging topic. Currently, numerical methods for solving the radiative transfer equation can be categorized into two groups: probabilistic approaches, for example, the direct simulation Monte Carlo methods [22, 13, 1], and the deterministic methods [4, 20, 9, 27, 2, 10]. The deterministic methods mainly include the long or short characteristic methods [7, 19], the discrete ordinate methods $\left(S_{N}\right)$ [15, 31], and the moment methods [32, 27].

The moment method is an effective approach to solve the radiative transfer equation, as moments are often the quantities we are interested in. For example, the radiation energy is the zeroth moment and the radiation energy flux is the first moment. However, the governing equation of each moment usually contains higher order moments, therefore an approximation for the higher order moments is required to complete the moment system. This is called the moment closure, which is the central problem of the moment method. Two important properties of the moment closure are hyperbolicity and realizability. Hyperbolicity is a necessary condition for the well-posedness of the first order moment systems, while realizability is a natural requirement that comes from the positivity of the underlying distribution function. However, the most commonly used moment model, the spherical harmonics $\left(P_{N}\right)$ method [28] does not possess realizability. Therefore, the result of the $P_{N}$ model sometimes leads to negative particle concentration [6, 5, 24]. In fact, for multidimensional cases, the maximum entropy model $\left(M_{N}\right)$ [9. 27] possesses both hyperbolicity and realizability. However, the closing relationship of the maximum entropy model is not explicit, making it too expensive to be applied in the numerical simulations.

In some cases, the dimension of the radiative transfer equation can be reduced due to symmetry considerations. One widely studied dimension-reduced case is that of slab geometry [28, 1, 2, 11]. For the slab geometry case, the distribution function varies only along one direction in spatial space and is an axisymmetric function in microscopic velocity space. Another case is the spherically symmetric case. The spherically symmetric case has many applications, such as in the studies of extended atmospheres [25], and there are many previous studies [12, 3, 17]. Compared to the radiative transfer equation in the slab geometry case, the transfer equation in spherical symmetry is more complicated due to the appearance of a derivative with respect to the microscopic velocity, which is not present in the slab geometry case.

The present paper is an extension of a model previously studied in [2] for the slab geometry case to the spherically symmetric case. The $B_{2}$ model studied in [2] is the second order case of the Extended Quadrature Method of Moments (EQMOM) [1]. For the slab geometry cases, the $B_{2}$ model gives an explicit closing relationship but possesses many of the essential properties of the attractive but 
much more expensive maximum entropy model. It was shown that the $B_{2}$ model is globally hyperbolic and possesses a non-negative ansatz for all realizable moments, and numerical simulation shows it has similar results as the $M_{2}$ model for benchmark problems in slab geometry cases.

In this work, we apply the ansatz of the $B_{2}$ model to the spherically symmetric case. Due to spherical symmetry, the distribution function should also be axisymmetric, which indicates that it is natural to extend the $B_{2}$ model to the spherically symmetric case. The resulting moment system is similar to the $B_{2}$ moment system in slab geometry in the convection term and possesses both global hyperbolicity and realizability. We also derive the connection condition between rarefaction and shock waves when there are simple wave conditions. Several numerical simulations are computed to validate the $B_{2}$ model in spherically symmetric cases and show the advantages of the $B_{2}$ model compared to other numerical methods.

The rest of this paper is organized as follows: In Section 2 we briefly introduce the RTE in the spherically symmetric case; then in Section 3 we introduce the $B_{2}$ model for spherical symmetry and analyze its properties. Numerical results are shown in Section 4 and the paper ends with a brief summary and conclusion in Section 5 .

\section{Preliminaries}

We consider the radiative transfer equation (RTE) in spherical symmetry [28]

$$
\frac{1}{c} \frac{\partial I}{\partial t}+\mu \frac{\partial I}{\partial r}+\frac{1-\mu^{2}}{r} \frac{\partial I}{\partial \mu}=\mathcal{C}(I)
$$

where

$$
\mathcal{C}(I)=-\sigma_{a} I-\sigma_{s} I+\frac{\sigma_{s}}{2} \int_{-1}^{1} I(\mu) \mathrm{d} \mu+\frac{S}{2} .
$$

Here $c$ is the speed of light, $I=I(t, r, \mu)$ is the specific intensity, $t \in \mathbb{R}^{+}$is time, $r \in\left[r_{L}, r_{R}\right] \subset \mathbb{R}^{+}$is the radial distance, and $\mu \in[-1,1]$ is the velocity related variable where $\arccos (\mu)$ represents the polar angle, i.e., the angle between the photon velocity and the radial distance $\mathbf{r}$. Also, $\sigma_{\mathrm{a}}$ and $\sigma_{\mathrm{s}}$ are the absorption and scattering coefficients, respectively, and $S$ is the external source term. Compared to the radiative transfer equation in slab geometry, which has the form

$$
\frac{1}{c} \frac{\partial I}{\partial t}+\mu \frac{\partial I}{\partial x}=\mathcal{C}(I)
$$

equation (1) is more complicated because of the presence of a derivative with respect to the polar angle, a term which does not appear in (3). 
We can obtain a moment system from equation (1) by taking its moments with respect to $\mu$, truncating the system and specifying a closure. Denote the $k$-th moment of the specific intensity as

$$
\langle I\rangle_{k} \triangleq \int_{-1}^{1} \mu^{k} I(\mu) \mathrm{d} \mu, \quad k \in \mathbb{N}
$$

we multiply equation (1) by $\mu^{k}$ and integrate over $\mu \in[-1,1]$ to get

$$
\frac{1}{c} \frac{\partial\langle I\rangle_{k}}{\partial t}+\frac{\partial\langle I\rangle_{k+1}}{\partial r}+\frac{(k+2)\langle I\rangle_{k+1}-k\langle I\rangle_{k-1}}{r}=\langle\mathcal{C}(I)\rangle_{k},
$$

where

$$
\langle\mathcal{C}(I)\rangle_{k}=-\left(\sigma_{a}+\sigma_{s}\right)\langle I\rangle_{k}+\frac{1+(-1)^{k}}{2 k+2}\left(\sigma_{s}\langle I\rangle_{0}+S\right) .
$$

The governing equation of $\langle I\rangle_{k}$ in (5) depends on higher order moment $\langle I\rangle_{k+1}$, indicating that the full system contains an infinite number of equations. For a given integer $N \in \mathbb{N}$, we truncate the system and only keep the governing equations of $\langle I\rangle_{k}, k \leq N$. However, the first $N+1$ equations contain $N+2$ unknown variables; thus the truncated system is not closed. In order to obtain a closed system, we have to approximate the $(N+1)$-th moment $\langle I\rangle_{N+1}$ based on the first $N+1$ moments $\langle I\rangle_{k}, k=0, \cdots, N$, which is formulated as

$$
\langle I\rangle_{N+1} \approx E_{N+1}=E_{N+1}\left(\langle I\rangle_{0},\langle I\rangle_{1}, \cdots,\langle I\rangle_{N}\right),
$$

and this is called the moment closure. A popular way to obtain the moment closure is to construct a specific intensity $\hat{I}$ based on the known moments. Let $E_{k}$, $k=0,1, \cdots, N$, be the known moments of a specific intensity $I$, we can construct a specific intensity $\hat{I}\left(\mu ; E_{0}, E_{1}, \cdots, E_{N}\right)$, which is called the ansatz of $I$, to approximate the unknown specific intensity $I$, such that,

$$
\int_{-1}^{1} \mu^{k} \hat{I}\left(\mu ; E_{0}, E_{1}, \cdots, E_{N}\right) \mathrm{d} \mu=E_{k}, \quad k=0,1, \cdots, N .
$$

Then the $(N+1)$-th moment can be directly approximated by the $(N+1)$-th moment of $\hat{I}$,

$$
E_{N+1}=\int_{-1}^{1} \mu^{N+1} \hat{I}\left(\mu ; E_{0}, E_{1}, \cdots, E_{N}\right) \mathrm{d} \mu,
$$

which closes the moment system (5).

The physically relevant value of a given set of moments for the RTE should satisfy constraints imposed by the positivity of the specific intensity. It is referred to as the realizable region. Realizability of the closing relationship ensures the closure is consistent with our apriori knowledge about the moments of a probability distribution function and is expected to improve the accuracy of the truncated system of equations [16]. Below we recall the definition of the realizable region. 
Definition 1. The realizable region for the first $N+1$ moments for any given integer $N \in \mathbb{N}$, denoted as $\Omega_{R, N}$, is the set of moments where each point corresponds to a positive specific intensity,

$$
\Omega_{R, N} \triangleq\left\{\left(E_{0}, E_{1}, \cdots, E_{N}\right)^{T}: \exists I(\mu)>0,\langle I\rangle_{k}=E_{k}, k=0,1, \cdots, N\right\} .
$$

The focus of this paper is to find a closing relationship of the second order moment model, for which the known moments are in the realizable region, which we will study in the next section.

\section{$3 \quad B_{2}$ Model for Spherical Symmetry}

\subsection{The $B_{2}$ closure}

The moment model for $N=2$ is written as

$$
\frac{1}{c} \frac{\partial \mathbf{E}}{\partial t}+\frac{\partial \mathbf{F}}{\partial r}=\mathbf{C}
$$

where $\mathbf{E}=\left(E_{0}, E_{1}, E_{2}\right)^{T}, \mathbf{F}=\left(E_{1}, E_{2}, E_{3}\right)^{T}$, and

$$
\mathbf{C}_{k}=-\frac{(k+2) E_{k+1}-k E_{k-1}}{r}-\sigma_{t} E_{k}+\frac{1+(-1)^{k}}{2 k+2}\left(\sigma_{s} E_{0}+S\right), \quad k=0,1,2 .
$$

The realizable region for $\left(E_{0}, E_{1}, E_{2}\right)$ can be derived from the Cauchy-Schwarz inequality to be

$$
\Omega_{R, 2}=\left\{\left(E_{0}, E_{1}, E_{2}\right)^{T}: E_{0}>0, E_{2}<E_{0}, E_{1}^{2}<E_{0} E_{2}\right\} .
$$

To close system (8), we use the following beta distribution ansatz,

$$
\hat{I}_{B_{2}}\left(\mu ; E_{0}, E_{1}, E_{2}\right)=\frac{w}{2 \mathbf{B}(\xi, \eta)}\left(\frac{1+\mu}{2}\right)^{\xi-1}\left(\frac{1-\mu}{2}\right)^{\eta-1}, \xi=\frac{\gamma}{\delta}, \eta=\frac{1-\gamma}{\delta},
$$

with $w>0,0<\gamma<1, \delta>0$. The ansatz (10) has the same form as the $B_{2}$ ansatz for slab geometry previously studied in [2]. Consistency of the known moments (7) determines the parameters $w, \gamma$, and $\delta$, as

$$
w=E_{0}, \quad \gamma=\frac{E_{1} / E_{0}+1}{2}, \quad \text { and } \quad \delta=\frac{\left(E_{1} / E_{0}\right)^{2}-E_{2} / E_{0}}{E_{2} / E_{0}-1}
$$

giving the moment closure

$$
E_{3}=\int_{-1}^{1} \mu^{3} \hat{I}_{B_{2}} \mathrm{~d} \mu=\frac{E_{1}\left(E_{2}^{2}+2 E_{1}^{2}-3 E_{0} E_{2}\right)}{2 E_{1}^{2}-E_{0} E_{2}-E_{0}^{2}} .
$$


System (8) with the closing relationship (12) shall be referred to as the $B_{2}$ model for spherical symmetry hereafter, and it differs from that studied in [2] in that it contains curvature terms brought about by spherical geometry.

In [2] it was proved that the $B_{2}$ model for slab geometry is globally hyperbolic, and there exists a non-negative ansatz for realizable moments. Hyperbolicity is necessary for the well-posedness of the Cauchy initial value problem. Moreover, the existence of a non-negative ansatz ensures the realizability of the closure. System (8) has the same convection terms as the $B_{2}$ system for slab geometry studied in [2], therefore it is also globally hyperbolic and realizable.

Next, we study the Riemann problem because its solution structure is instructive for the design of the approximate Riemann solver, which is the basis of the numerical methods using Godunov-type schemes. Note that this problem has not yet been studied for the slab geometry case, and the analysis below can also be applied to that scenario.

\subsection{Riemann problem}

Define $\mathbf{E}=\left(E_{0}, E_{1}, E_{2}\right)^{T}$ and $\mathbf{F}=\left(E_{1}, E_{2}, E_{3}\right)^{T}$, where $E_{3}$ is given by (12). Consider the following Riemann problem:

$$
\begin{aligned}
& \frac{1}{c} \frac{\partial \mathbf{E}}{\partial t}+\frac{\partial \mathbf{F}}{\partial r}=0, \\
& \mathbf{E}(0, r)=\left\{\begin{array}{l}
\mathbf{E}^{L}, r<r_{0}, \\
\mathbf{E}^{R}, r>r_{0} .
\end{array}\right.
\end{aligned}
$$

We first recall results from [2] in two lemmas which will be used in the following discussions in this section. Hereafter, the eigenvalues and eigenvectors of the Jacobian matrix

$$
\boldsymbol{J}=\frac{\partial \mathbf{F}}{\partial \mathbf{E}}=\left(\begin{array}{ccc}
0 & 1 & 0 \\
0 & 0 & 1 \\
\frac{\partial E_{3}}{\partial E_{0}} & \frac{\partial E_{3}}{\partial E_{1}} & \frac{\partial E_{3}}{\partial E_{2}}
\end{array}\right),
$$

are denoted by $\lambda_{k}$ and $\mathbf{r}_{k}, k=1,2,3$, with $\lambda_{1}<\lambda_{2}<\lambda_{3}$, and we also define $\Delta_{k} \triangleq \nabla_{\mathbf{E}} \lambda_{k} \cdot \mathbf{r}_{k}$, which is essential to analyze the characteristic structure.

Lemma 3.1. For any $\mathbf{E} \in \Omega_{R, 2}$, we have $-1<\lambda_{1}<\frac{E_{1}}{E_{0}}<\lambda_{3}<1$.

Moreover, the eigenvector of $\mathbf{J}$ with respect to the eigenvalue $\lambda_{k}$ is $\mathbf{r}_{k}=$ $\left(1, \lambda_{k}, \lambda_{k}^{2}\right)^{T}$. 
Lemma 3.2. $\Delta_{1}<0$ and $\Delta_{3}>0$ hold for any $\mathbf{E} \in \Omega_{R, 2}$. However, $\Delta_{2}$ changes sign over $\Omega_{R, 2}$.

It was proved in [2] that the first and third characteristic fields are genuinely nonlinear, while the second characteristic field is neither genuinely nonlinear nor linearly degenerate. This indicates the solution of the Riemann problems may contain rather complex behavior. However, we would still expect simple characteristic waves in the solution of Riemann problems for first and third waves. Simple wave solutions of the above Riemann problems are the composition of one intermediate state connected by rarefaction waves or shock waves. In order to get full understanding of the system, these waves will be studied respectively below.

To simplify discussions, we denote

$$
u=\frac{E_{1}}{E_{0}}, \quad p=E_{2}-\frac{E_{1}^{2}}{E_{0}} .
$$

Rarefaction waves We first investigate the integral curves given by rarefaction waves. We take the first characteristic field as an example. Suppose that two states $\mathbf{E}^{L}=\left(E_{0}^{L}, E_{1}^{L}, E_{2}^{L}\right)^{T}$ and $\mathbf{E}^{R}=\left(E_{0}^{R}, E_{1}^{R}, E_{2}^{R}\right)^{T}$ are connected by a rarefaction wave corresponding to the first characteristic field. Take $\zeta$ as the parameter such that $\tilde{\mathbf{E}}(0)=\mathbf{E}^{L}$ and $\tilde{\mathbf{E}}\left(\zeta_{*}\right)=\mathbf{E}^{R}$, while

$$
\tilde{\mathbf{E}}(\zeta)=\left(\tilde{E}_{0}(\zeta), \tilde{E}_{1}(\zeta), \tilde{E}_{2}(\zeta)\right)^{T}
$$

denotes the integral curve in the 3-dimensional phase space. The corresponding eigenvalues and eigenvectors are denoted as $\tilde{\lambda}_{1}(\zeta)$ and $\tilde{\mathbf{r}}_{1}(\zeta)$.

According to the theory of integral curves of rarefaction waves [21, Chapter 13.8.1], there exists a nonzero scalar factor $\tilde{\alpha}(\zeta)$, such that

$$
\tilde{\mathbf{E}}^{\prime}(\zeta)=\tilde{\alpha}(\zeta) \tilde{\mathbf{r}}_{1}(\tilde{\mathbf{E}}(\zeta))
$$

Recalling $\tilde{\mathbf{r}}_{1}=\left(1, \tilde{\lambda}_{1}, \tilde{\lambda}_{1}^{2}\right)^{T}$, by direct calculation we obtain from (16)

$$
\frac{\mathrm{d} \tilde{E}_{0}(\zeta)}{\mathrm{d} \zeta}=\tilde{\alpha}(\zeta), \quad \frac{\mathrm{d} \tilde{u}(\zeta)}{\mathrm{d} \zeta}=\tilde{\alpha}(\zeta)\left(\tilde{\lambda}_{1}-\tilde{u}\right), \quad \frac{\mathrm{d} \tilde{p}(\zeta)}{\mathrm{d} \zeta}=\tilde{\alpha}(\zeta)\left(\tilde{\lambda}_{1}-\tilde{u}\right)^{2}
$$

Combine the entropy condition $\tilde{\lambda}_{1}(0)=\lambda_{1}^{L}<\lambda_{1}^{R}=\tilde{\lambda}_{1}\left(\zeta_{*}\right)$ with

$$
\frac{1}{\tilde{\alpha}(\zeta)} \frac{\mathrm{d} \tilde{\lambda_{1}}(\zeta)}{\mathrm{d} \zeta}=\frac{1}{\tilde{\alpha}(\zeta)} \nabla_{\tilde{\mathbf{E}}} \tilde{\lambda}_{1} \cdot \frac{\mathrm{d} \tilde{\mathbf{E}}}{\mathrm{d} \zeta}=\nabla_{\tilde{\mathbf{E}}} \tilde{\lambda}_{1} \cdot \tilde{\mathbf{r}}_{1}<0,
$$


where the last inequality holds because of Lemma 3.2, we could see that $\tilde{\alpha}\left(\zeta_{*}\right) \zeta_{*}<$ 0 . Also, according to Lemma 3.1 we have $\tilde{\lambda}_{1}<\tilde{u}$, therefore (17) indicates that

$$
E_{0}^{L}>E_{0}^{R}, \quad u^{L}<u^{R}, \quad p^{L}>p^{R} .
$$

Analogously, for the third wave, we have

$$
E_{0}^{L}<E_{0}^{R}, \quad u^{L}<u^{R}, \quad p^{L}<p^{R} .
$$

Shock waves We consider shock waves by taking the first characteristic field as an example. Suppose $\mathbf{E}^{L}=\left(E_{0}^{L}, E_{1}^{L}, E_{2}^{L}\right)^{T}$ and $\mathbf{E}^{R}=\left(E_{0}^{R}, E_{1}^{R}, E_{2}^{R}\right)^{T}$ are connected by a shock corresponding to the first characteristic field, then according to the Rankine-Hugoniot condition, there exists $s_{1} \in \mathbb{R} \backslash\{0\}$, such that

$$
\begin{aligned}
& E_{1}^{R}-E_{1}^{L}=s_{1}\left(E_{0}^{R}-E_{0}^{L}\right), \\
& E_{2}^{R}-E_{2}^{L}=s_{1}\left(E_{1}^{R}-E_{1}^{L}\right), \\
& E_{3}^{R}-E_{3}^{L}=s_{1}\left(E_{2}^{R}-E_{2}^{L}\right) .
\end{aligned}
$$

From the first two equations of (19),

$$
s_{1}=\frac{E_{1}^{R}-E_{1}^{L}}{E_{0}^{R}-E_{0}^{L}}=\frac{E_{2}^{R}-E_{2}^{L}}{E_{1}^{R}-E_{1}^{L}} .
$$

Direct calculations from the above equalities yield

$$
\begin{aligned}
& \left(E_{0}^{R}-E_{0}^{L}\right)\left(u^{R}-u^{L}\right)=\frac{\left(E_{0}^{R}-E_{0}^{L}\right)^{2}}{E_{0}^{R}}\left(s_{1}-u^{L}\right), \\
& \left(E_{0}^{R}-E_{0}^{L}\right)\left(p^{R}-p^{L}\right)=\frac{1}{E_{0}^{L} E_{0}^{R}}\left(E_{0}^{R} E_{1}^{L}-E_{0}^{L} E_{1}^{R}\right)^{2}>0 .
\end{aligned}
$$

Also, according to the entropy condition and Lemma 3.1 .

$$
u^{L}>\lambda_{k}^{L}>s_{1}>\lambda_{k}^{R},
$$

indicating $\left(E_{0}^{R}-E_{0}^{L}\right)\left(u^{R}-u^{L}\right)<0$. Therefore, the signs of $u^{R}-u^{L}$ and $p^{R}-p^{L}$ are determined by sign of $E_{0}^{R}-E_{0}^{L}$.

Next, we consider the sign of $E_{0}^{R}-E_{0}^{L}$. Parameterize the Hugoniot curve as $\tilde{\mathbf{E}}(\tau)$ with $\tilde{\mathbf{E}}(0)=\mathbf{E}^{L}$ and $\tilde{\mathbf{E}}(1)=\mathbf{E}^{R}$. For any $0 \leq \tau_{*}<\tau_{*}+\varepsilon \leq 1$ define $\mathbf{E}^{l}=\tilde{\mathbf{E}}\left(\tau_{*}\right)$ and $\mathbf{E}^{r}=\tilde{\mathbf{E}}\left(\tau_{*}+\varepsilon\right)$, then $\mathbf{E}^{l}$ and $\mathbf{E}^{r}$ satisfy the Rankine-Hugoniot condition (19) and the entropy condition (22), therefore also (21). Assuming $\varepsilon$ is sufficiently small, then according to (19), we have

$$
E_{0}^{r}=E_{0}^{l}+d, \quad E_{1}^{r}=E_{1}^{l}+s_{1} d, \quad E_{2}^{r}=E_{2}^{l}+s_{1}^{2} d, \quad E_{3}^{r}=E_{3}^{l}+s_{1}^{3} d,
$$


where $|d| \ll 1$, and $\lambda_{1}^{l}>s_{1}>\lambda_{1}^{r}$. As $\lambda_{1}$ is a smooth function of $\mathbf{E}$, Taylor expansion yields

$$
\lambda_{1}^{r}-\lambda_{1}^{l}=\lambda_{1}\left(\mathbf{E}^{r}\right)-\lambda_{1}\left(\mathbf{E}^{l}\right)=d\left(\left(\frac{\partial \lambda_{1}}{\partial E_{0}}\right)^{l}+\left(\frac{\partial \lambda_{1}}{\partial E_{1}}\right)^{l} s_{1}+\left(\frac{\partial \lambda_{1}}{\partial E_{2}}\right)^{l} s_{1}^{2}\right)+O\left(d^{2}\right) .
$$

According to Lemma 3.2, we have

$$
\left(\frac{\partial \lambda_{1}}{\partial E_{0}}\right)^{l}+\left(\frac{\partial \lambda_{1}}{\partial E_{1}}\right)^{l} \lambda_{1}^{l}+\left(\frac{\partial \lambda_{1}}{\partial E_{2}}\right)^{l}\left(\lambda_{1}^{l}\right)^{2}=\Delta_{1}<0
$$

Notice that $|d| \ll 1$ indicates $\left|\lambda_{1}^{r}-\lambda_{1}^{l}\right| \ll 1$, thus $\left|s_{1}-\lambda_{1}^{l}\right| \ll 1$. Therefore, when $|d| \ll 1$,

$$
\left(\frac{\partial \lambda_{1}}{\partial E_{0}}\right)^{l}+\left(\frac{\partial \lambda_{3}}{\partial E_{1}}\right)^{l} s_{1}+\left(\frac{\partial \lambda_{1}}{\partial E_{2}}\right)^{l}\left(s_{1}\right)^{2}<0 .
$$

Combining (23) and (25) with entropy condition (22), we obtain

$$
d>0, \quad E_{0}^{r}>E_{0}^{l}, \quad u^{r}<u^{l}, \quad p^{r}>p^{r} .
$$

Notice that (26) holds for any $\tau_{\star} \in[0,1)$, and according to the finite covering theorem, we can obtain the following results for the first characteristic field.

$$
E_{0}^{R}>E_{0}^{L}, \quad u^{R}<u^{L}, \quad p^{R}>p^{L} .
$$

For the third characteristic field, we analogously have

$$
E_{0}^{R}<E_{0}^{L}, \quad u^{R}<u^{L}, \quad p^{R}<p^{L} .
$$

Before the end of this subsection, we summarize the discussion on the entropy condition of two types of waves in the following theorem.

Theorem 3.1. For the $B_{2}$ moment system, the waves of the first and third families are elementary, and their types can be determined by the sign of $\lambda-u$ and the macroscopic parameters on the both sides of the waves:

\section{Numerical Experiments}

In this section, we discuss the numerical scheme for the $B_{2}$ model for spherical symmetry and perform numerical simulations on some typical examples to verify its validity. In all subsequent computation we assume $c=1$ in equation (8). 


\begin{tabular}{|r|c|c|c|}
\hline & $E_{0}$ & $u$ & $p$ \\
\hline \multirow{2}{*}{ Rarefaction wave } & 1-wave $E_{0}^{L}>E_{0}^{R}$ & \multirow{2}{*}{$u^{L}<u^{R}$} & 1-wave $p^{L}>p^{R}$ \\
\cline { 2 - 2 } & 3-wave $E_{0}^{L}<E_{0}^{R}$ & & 3-wave $p^{L}<p^{R}$ \\
\hline \multirow{2}{*}{ Shock wave } & 1-wave $E_{0}^{L}<E_{0}^{R}$ & \multirow{2}{*}{$u^{L}>u^{R}$} & 1-wave $p^{L}<p^{R}$ \\
& 3-wave $E_{0}^{L}>E_{0}^{R}$ & & 3-wave $p^{L}>p^{R}$ \\
\hline
\end{tabular}

Table 1: wave structure of one dimension $B_{2}$ model

\subsection{Numerical scheme}

We use an equidistant discretization of $N_{\text {cell }}$ cells for the spatial domain $\left[r_{L}, r_{R}\right]$, where $0 \leq r_{L}<r_{R}$. Therefore, the spatial step is $\Delta r=\frac{r_{R}-r_{L}}{N_{\text {cell }}}$, with grid points $r_{i}=r_{L}+(i-1 / 2) \Delta r, i=1, \cdots, N_{\text {cell }}$, and cells $\left[r_{i-1 / 2}, r_{1+1 / 2}\right], i=1, \cdots, N_{\text {cell }}$, with midpoints $r_{1-1 / 2}=r_{i}-\Delta r / 2$. We denote the approximation of the solution and the source term in the $i$-th cell at time step $t_{n}$ as $\mathbf{E}_{i}^{n}$ and $\mathbf{C}_{i}^{n}$, respectively.

We adopt the splitting scheme described in [21, Chapter 17.6]. Problem (8) is split into a hyperbolic conservation law, $\frac{\partial \mathbf{E}}{\partial t}+\frac{\partial \mathbf{F}}{\partial r}=0$, and a simple ODE, $\frac{\partial \mathbf{E}}{\partial t}=\mathbf{C}$. The former is solved under the finite volume framework, and the latter is solved using the backward Euler method. The numerical scheme is formulated as

$$
\begin{aligned}
& \frac{\mathbf{E}_{i}^{*}-\mathbf{E}_{i}^{n}}{\Delta t}+\frac{\mathbf{F}_{i+1 / 2}-\mathbf{F}_{i-1 / 2}}{\Delta r_{i}}=0, \\
& \frac{\mathbf{E}_{i}^{n+1}-\mathbf{E}_{i}^{*}}{\Delta t}=\mathbf{C}_{i}^{n+1},
\end{aligned}
$$

where $\mathbf{E}_{i}^{*}$ is the intermediate value in the $i$-th cell between the time step $t_{n}$ and $t_{n+1}, \mathbf{F}_{i+1 / 2}$ is the numerical flux at $r=r_{i+1 / 2}$, and the $k$-th entry of the source term $\mathbf{C}_{i}^{n+1}$ is

$$
C_{k, i}^{n+1}=-\frac{(k+2) E_{k+1, i}^{n+1}-k E_{k-1, i}^{n+1}}{r_{i}}-\sigma_{t, i}^{n+1} E_{k, i}^{n+1}+\frac{1+(-1)^{k}}{2 k+2}\left(\sigma_{s, i}^{n+1} E_{0, i}^{n+1}+S_{i}^{n+1}\right) .
$$

We compute $\mathbf{F}_{i+1 / 2}$ using the Lax-Friedrich flux and solve the nonlinear system resulting from the implicit discretization of the source term by the Newton iteration method.

The time step is constrained by the CFL condition

$$
\Delta t=\mathrm{CFL} \cdot \min _{i} \frac{\Delta r}{\max _{k}\left|\lambda_{k}\left(\mathbf{E}_{i}^{n}\right)\right|},
$$

where $\lambda_{k}\left(\mathbf{E}_{i}^{n}\right)$ is the $k$-th characteristic value of the $B_{2}$ model when the given moments are $\mathbf{E}_{i}^{n}$. Notice that it is proved in [2] that the characteristic speed of the 
$B_{2}$ model is not larger than 1 . Hence we take $\Delta t=\mathrm{CFL} \cdot \Delta r$, and the CFL number is chosen to be 0.95 .

In our numerical scheme, we also need to specify the values of $E_{1}, E_{2}$, and $E_{3}$ on the boundaries, which we derive from the kinetic level. Take the left boundary as an example. We define the flux vector $\hat{\mathbf{f}}^{\mathrm{L}}:=\left(E_{1}^{\mathrm{L}}, E_{1}^{\mathrm{L}}, E_{3}^{\mathrm{L}}\right)^{T}$ on the left boundary as

$$
E_{j}^{\mathrm{L}}=\int_{0}^{1} \mu^{j} I_{\text {out }} d \mu+\int_{-1}^{0} \mu^{j} \hat{I}_{B_{2}} d \mu, \quad \text { for } j=1,2,3,
$$

where $\hat{I}_{B_{2}}$ is the ansatz associated with the moment vector from the first spatial cell on the inner side of the boundary and $I_{\text {out }}$ is the distribution function outside of the domain depending on $\hat{I}_{B_{2}}$ and the actual boundary condition of the problem. For instance, for the vacuum boundary condition,

$$
I_{\text {out }}\left(t, r_{L}, \mu\right)=0, \quad \mu>0
$$

while for the reflective boundary condition,

$$
I_{\text {out }}\left(t, r_{L}, \mu\right)=\hat{I}_{B_{2}}\left(t, r_{L},-\mu\right), \quad \mu>0 .
$$

We use the reflective boundary condition on the left when $r_{L}=0$. Analogously, we can give the boundary condition for the right boundary at $r_{R}>0$.

\subsection{Numerical results}

Example 4.1 (Bilateral beams). This example is used to test the ability of the $B_{2}$ model to deal with anisotropic specific intensity. In this test, the spatial domain is unbounded (but $[0,1]$ for numerical experiments), $\sigma_{\mathrm{a}}$ and $\sigma_{\mathrm{s}}$ are set to 0 , and the external source term $S=0$. The initial state is taken as

$$
I(0, r, \mu)=\left\{\begin{array}{lr}
\frac{1}{r^{2}} \delta(\mu-1), & 0.2 \leq r \leq 0.4, \\
\frac{1}{r^{2}} \delta(\mu+1), & 0.7 \leq r \leq 0.9, \\
0, & \text { otherwise. }
\end{array}\right.
$$

The analytical solution of this problem is

$$
I(t, r, \mu)= \begin{cases}\frac{(r-t)^{2}}{r^{2}} I(0, r-t, 1), & \mu=1, \\ \frac{(r+t)^{2}}{r^{2}} I(0, r+t,-1), & \mu=-1 .\end{cases}
$$

Therefore, the analytical solutions of $E_{0}, E_{1}$ and $E_{2}$ are

$$
E_{0}(t, r)=E_{2}(t, r)=\frac{1}{r^{2}} \chi_{[0.2+t, 0.4+t]}(r)+\frac{1}{r^{2}} \chi_{[0.7-t, 0.9-t]}(r),
$$




$$
E_{1}(t, r)=\frac{1}{r^{2}} \chi_{[0.2+t, 0.4+t]}(r)-\frac{1}{r^{2}} \chi_{[0.7-t, 0.9-t]}(r),
$$

where $\chi_{A}(r)$ is the indicator function, defined as

$$
\chi_{A}(r)= \begin{cases}1, & r \in A \\ 0, & r \notin A\end{cases}
$$

This problem describes two shells. The inner shell spreads out while the outer shell contracts to the center. The specific intensities of these two shells are delta functions. When the two shells intersect, the specific intensity is the sum of two delta functions $\delta(\mu-1)$ and $\delta(\mu+1)$. This kind of highly anisotropic specific intensity is difficult to approximate by traditional methods [11].

However, when the actual distribution function is the sum of two delta functions,

$$
I=C_{1} \delta(\mu-1)+C_{2} \delta(\mu+1)
$$

where $C_{1}$ and $C_{2}$ are constants. The moments of the distribution function in (30) are $E_{0}=E_{2}=C_{1}+C_{2}$ and $E_{1}=C_{1}-C_{2}$. According to the closure (12), we have that the moment closure given by the $B_{2}$ model is

$$
E_{3}=C_{1}-C_{2},
$$

which is the third moment of the actual distribution function. Therefore, the $B_{2}$ model can deal with this kind of highly anisotropic distribution function.

We simulate this problem with $N_{\text {cell }}=10000$ cells until $t_{\text {end }}=0.1,0.2,0.25,0.3,0.4$, and the results of $E_{0}, E_{1}$, and $E_{2}$ are shown in Figure 1, 2, and 3 , respectively.

Figure 1 demonstrates that the $B_{2}$ model can obtain an accurate solution of $E_{0}$ at all times. When $t=0$ and 0.1 , the two waves are independent; when $t=$ $0.2,0.25$, and 0.3 , the two waves intersect each other; when $t=0.4$, the two waves separate. Also, according to the results in Figure 2 and Figure 3 , the $B_{2}$ solutions give first and second order moments which are almost identical with the exact solution. These results indicate that the $B_{2}$ model can describe this highly anisotropic specific intensity.

We use the $P_{N}$ method with $N=10,30$, and 70 to do the same simulations, and the results of $E_{0}$ at $t=0.1$ are presented in Figure 4. The $P_{N}$ solution of $N=10$ and 30 are oscillatory and contains negative values for $E_{0}$, showing that the $P_{N}$ method can not approximate this kind of beam-like distribution well. As $N$ gets larger, the oscillation decreases in magnitude. However, the $P_{N}$ method requires to take as many as $N=70$ to get a sufficiently satisfying result.

Example 4.2 (Laser beams propagating into vacuum). We use the same setup as Example 4.1 where the opacities $\sigma_{a}=\sigma_{s}=0$, and the external source term $S=0$. 


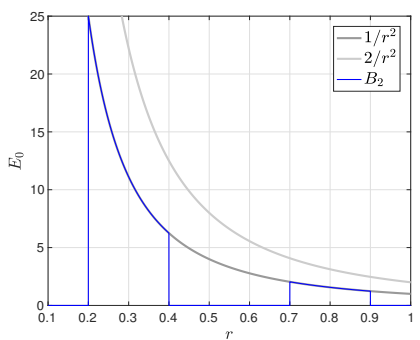

(a) solution of $E_{0}$ at $t=0$.

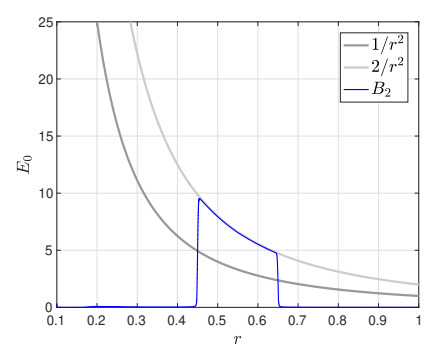

(d) solution of $E_{0}$ at $t=0.25$.

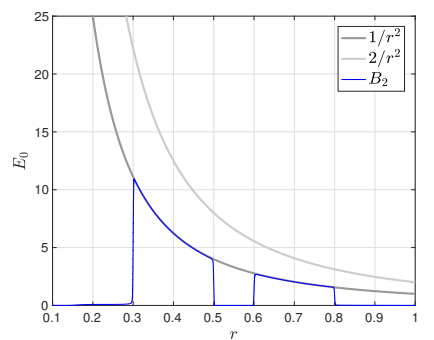

(b) solution of $E_{0}$ at $t=0.1$.

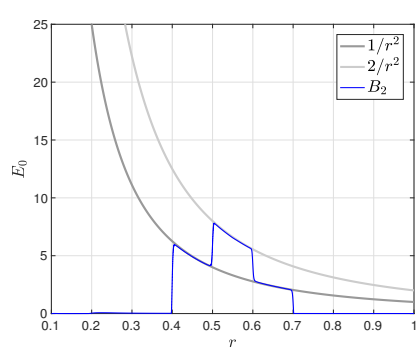

(e) solution of $E_{0}$ at $t=0.3$.

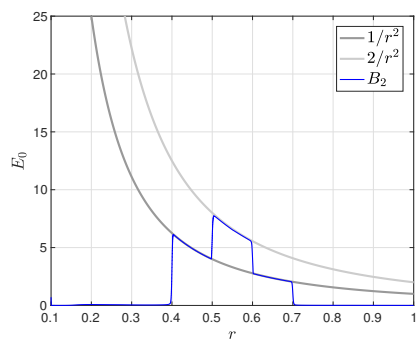

(c) solution of $E_{0}$ at $t=0.2$.

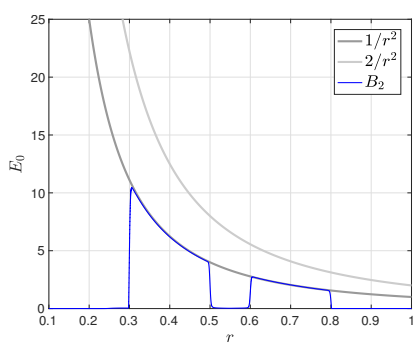

(f) solution of $E_{0}$ at $t=0.4$.

Figure 1: Numerical results of the $B_{2}$ model of $E_{0}$ for bilateral beams
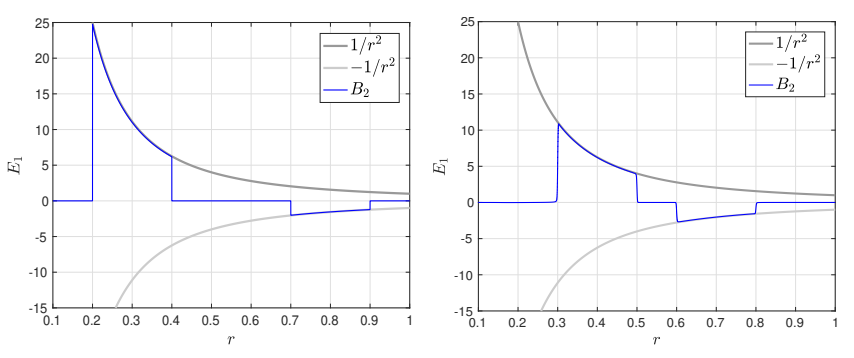

(b) solution of $E_{1}$ at $t=0.1$.
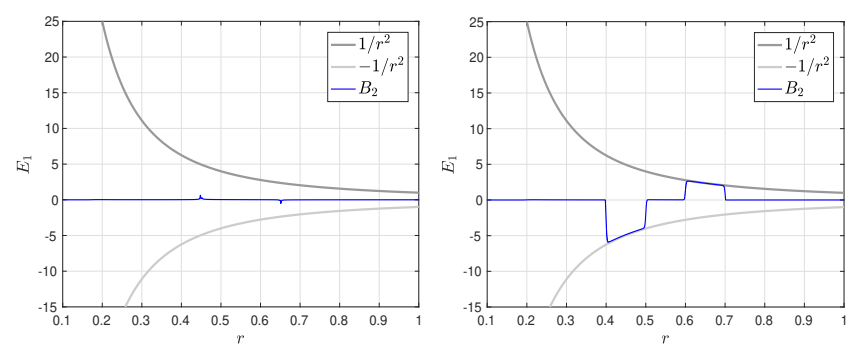

(e) solution of $E_{1}$ at $t=0.3$. (f) solution of $E_{1}$ at $t=0.4$.

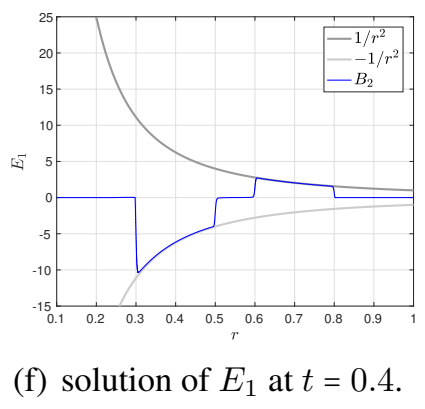

Figure 2: Numerical results of the $B_{2}$ model of $E_{1}$ for bilateral beams 


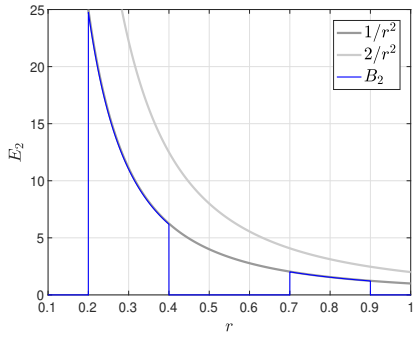

(a) solution of $E_{1} 2$ at $t=0$.

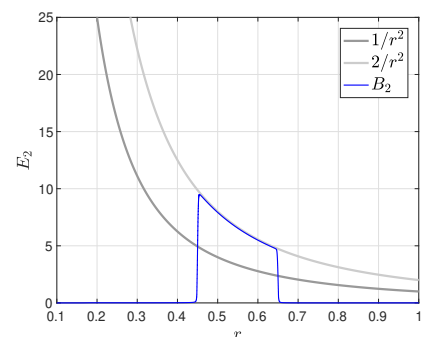

(d) solution of $E_{2}$ at $t=0.25$.

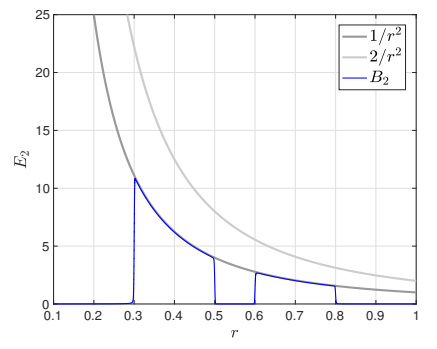

(b) solution of $E_{2}$ at $t=0.1$.

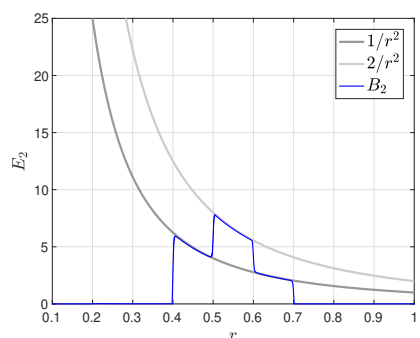

(e) solution of $E_{2}$ at $t=0.3$.

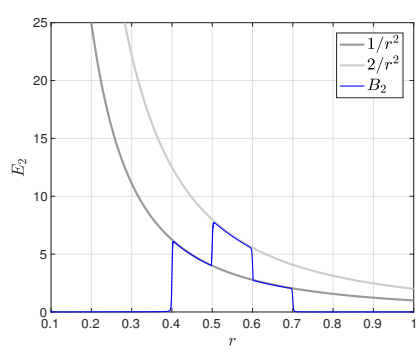

(c) solution of $E_{2}$ at $t=0.2$.

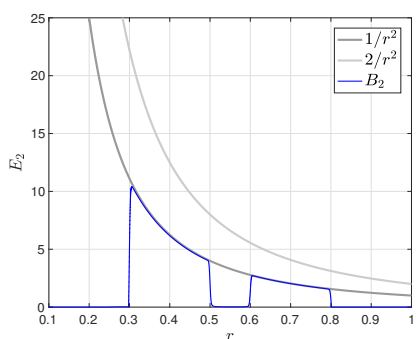

Figure 3: Numerical results of the $B_{2}$ model of $E_{2}$ for the bilateral beams

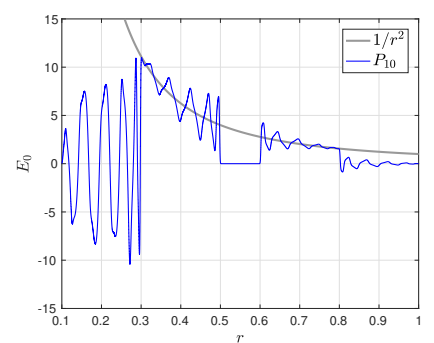

(a) Solution of $P_{10}$

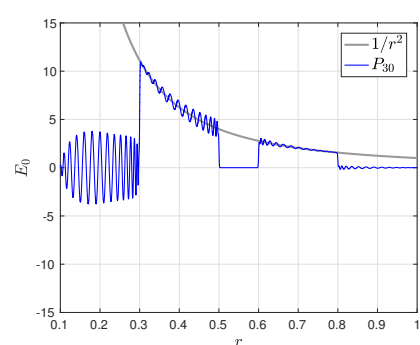

(b) Solution of $P_{30}$.

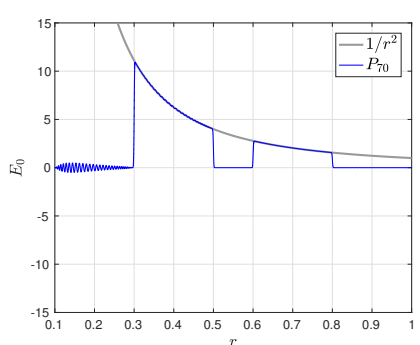

(c) Solution of $P_{70}$.

Figure 4: Numerical results of the $P_{N}$ method for the bilateral beams 
The domain is unbounded (but $[0,10]$ for numerical experiments). The initial state is taken as

$$
I(0, r, \mu)=\left\{\begin{array}{lr}
\frac{1}{r^{2}} \delta(\mu-(r-5)), & 4 \leq r \leq 6 \\
0, & \text { otherwise } .
\end{array}\right.
$$

The analytical solution can be obtained in the same way as in Example 4.1. Direct calculation shows the exact solution of this example to be

$$
I(t, r, \mu)=\left\{\begin{array}{lr}
\frac{1}{r^{2}(1+t)} \delta\left(\mu-\frac{r-5}{1+t}\right), & 4-t \leq r \leq 6+t \\
0, & \text { otherwise }
\end{array}\right.
$$

In this problem, every point corresponds to a Dirac delta function, which means that we need to approximate $\delta\left(\mu-\mu_{0}\right)$ for any $\mu_{0} \in[-1,1]$, because that at $r=\mu_{0}+5,4 \leq \mu_{0} \leq 6$, the initial state is $\frac{1}{r^{2}} \delta\left(\mu-\mu_{0}\right)$. This is difficult for commonly used numerical method, such as the discrete-ordinates $\left(S_{N}\right)$ and the spherical harmonics $\left(P_{N}\right)$. However, consider the actual distribution function $I=\delta\left(\mu-\mu_{0}\right)$, we have that

$$
E_{0}=1, \quad E_{1}=\mu_{0}, \quad E_{2}=\mu_{0}^{2} .
$$

According to (12), we can obtain the moment closure given by the $B_{2}$ model:

$$
E_{3}=\frac{E_{1}\left(E_{2}^{2}+2 E_{1}^{2}-3 E_{0} E_{2}\right)}{2 E_{1}^{2}-E_{0} E_{2}-E_{0}^{2}}=\mu_{0}^{3},
$$

which is the third moment of the actual distribution function. Therefore, the $B_{2}$ model can approximate the Dirac delta function $\delta\left(\mu-\mu_{0}\right)$ for any $\mu \in[-1,1]$.

We use $N_{\text {cell }}=100000$ cells to simulate this problem until $t=0.5$, and the results of $E_{0}, E_{1}$, and $E_{2}$ are shown in Figure 5. Results in Figure 5 show that the solutions of all the three moments considered in the $B_{2}$ model agree with the reference solution, demonstrating the effectiveness of the $B_{2}$ model in approximating this test case where the distribution function contain the Dirac function $\delta\left(\mu-\mu_{0}\right)$. Moreover, we use the $P_{N}$ method with $N=10,30$, and 50 to do the same simulations, and the results of $E_{0}$ are shown in Figure 6 . There are many oscillations in the results of the $P_{N}$ model, and as many as $N=50$ are required in order to get a satisfying result. Therefore, the $P_{N}$ model is not effective for describing this kind of strongly anisotropic distribution.

Example 4.3 (Homogeneous sphere). This example consists of a static homogeneous and isothermal sphere of radius $R$ that radiates into vacuum. The radiation 


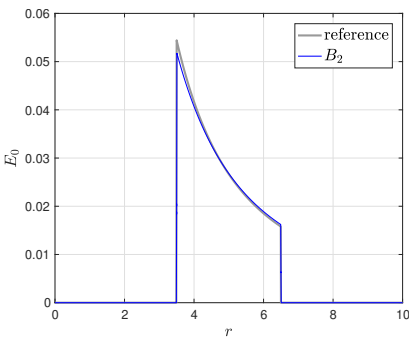

(a) Solution of $E_{0}$

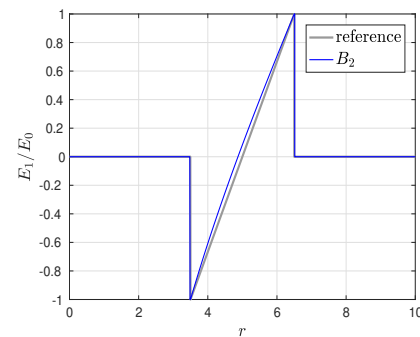

(b) Solution of $\frac{E_{1}}{E_{0}}$

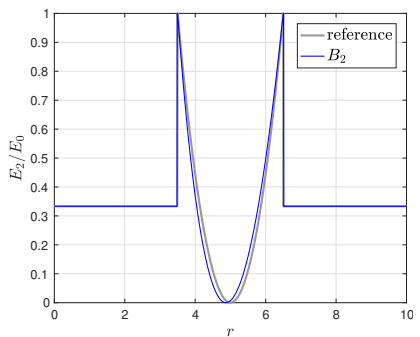

(c) Solution of $\frac{E_{2}}{E_{0}}$

Figure 5: Numerical results of the $B_{2}$ method for laser beams propagating into vacuum

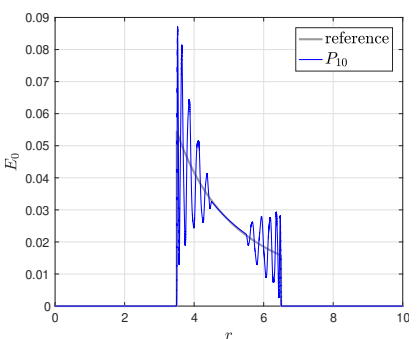

(a) Solution of $P_{10}$

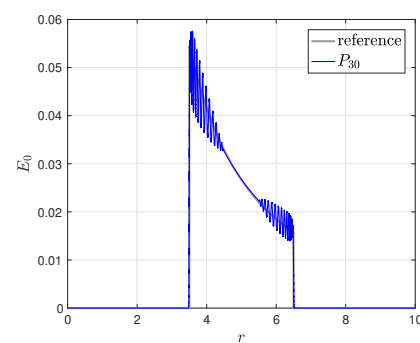

(b) Solution of $P_{30}$

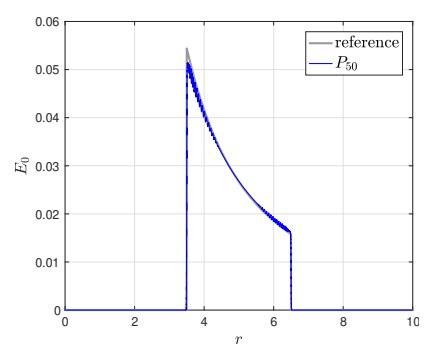

(c) Solution of $P_{50}$

Figure 6: Numerical results of the $P_{N}$ method for laser beams propagating into vacuum 
interacts with the background matter only via isotropic absorption and thermal emission inside the sphere.

$$
\mu \frac{\partial I}{\partial r}+\frac{1-\mu^{2}}{r} \frac{\partial I}{\partial \mu}= \begin{cases}\sigma_{a}(B-I), & r \leq R \\ 0, & r>R .\end{cases}
$$

The sphere radius is $R=1$ and the spatial domain is unbounded (but $[0,5]$ for numerical experiments). Inside the sphere the absorption and scattering opacities are chosen to be $\sigma_{a}=250$ and $\sigma_{s}=0$, respectively, and the emissivity $B=1$; while in the vacuum at $r>R$, we have $\sigma_{a}=\sigma_{s}=B=0$. The boundary condition at $r=5$ is an infinite boundary condition, and the initial state is $I(\mu)=0.5 \times 10^{-7}$ everywhere.

As this test case contains a sharp transition from the diffusive regime inside the sphere to the free-streaming regime outside, it is frequently employed to test radiative transfer codes [29, 30, 1].

The steady-state transport equation can be solved analytically [30]:

$$
I(r, \mu)=B\left(1-e^{-\sigma_{a} s(r, \mu)}\right),
$$

where

$$
s(r, \mu)= \begin{cases}r \mu+R g(r, \mu) & \text { if } r \leq R, \quad-1 \leq \mu \leq 1, \\ 2 R g(r, \mu) & \text { if } r>R, \quad \sqrt{1-\left(\frac{R}{r}\right)^{2}} \leq \mu \leq 1, \\ 0, & \text { otherwise }\end{cases}
$$

and

$$
g(r, \mu)=\sqrt{1-\left(\frac{r}{R}\right)^{2}\left(1-\mu^{2}\right)} .
$$

We use $N_{\text {cell }}=10000$ cells to calculate this example and the results of $E_{0}, E_{1}$, and $E_{2}$ of the $B_{2}$ model are shown in Figure 7. The results of the $P_{2}, P_{6}$, and $P_{10}$ model are shown in Figure 8 . The values of $\frac{E_{1}}{E_{0}}, \frac{E_{2}}{E_{0}}$, and $\frac{E_{2}}{E_{0}}-\left(\frac{E_{1}}{E_{0}}\right)^{2}$ in Figure 7 gives some indication as to the characteristics of the distribution function of this problem. First, we see that the distribution function of this numerical solution lies in the realizable region for any $r$. Second, when $r \leq R, \frac{E_{1}}{E_{0}}=0$ and $\frac{E_{2}}{E_{0}}=\frac{1}{3}$. This indicates that the distribution function $I(r, \mu)=\frac{E_{0}}{2}$, which is isotropic; when $r>R$, the results show that the distribution function gets more anisotropic when $r$ gets larger. When $r$ is large, according to the result of $\frac{E_{1}}{E_{0}}$ and $\frac{E_{2}}{E_{0}}-\frac{E_{1}^{2}}{E_{0}}$, we know that the distribution function is close to the boundary of the realizable region. The 


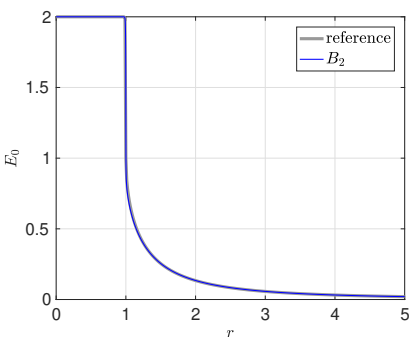

(a) Solution of $E_{0}$.

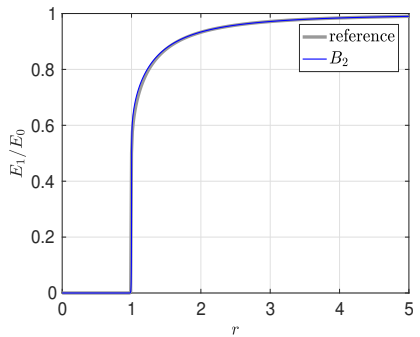

(d) Solution of $E_{1} / E_{0}$.

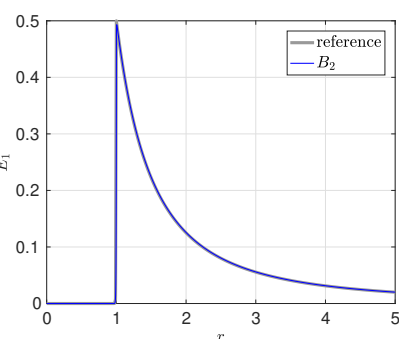

(b) Solution of $E_{1}$.

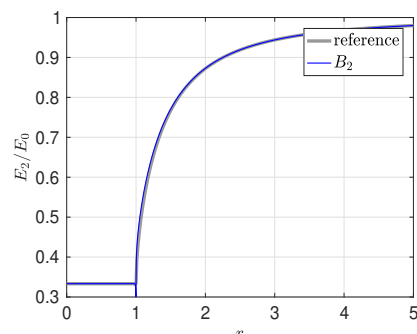

(e) Solution of $E_{2} / E_{0}$.

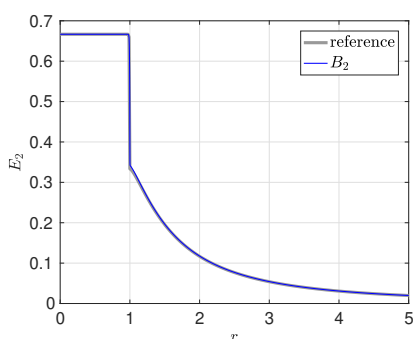

(c) Solution of $E_{2}$.

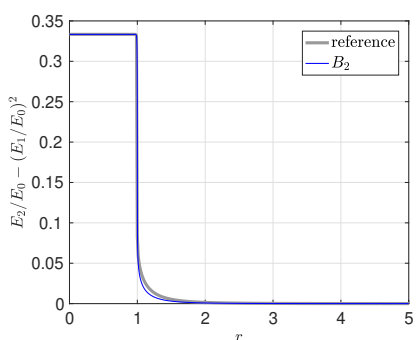

(f) Solution of $E_{2} / E_{0}-\left(E_{1} / E_{0}\right)^{2}$.

Figure 7: Numerical results of the $B_{2}$ model for homogeneous sphere example

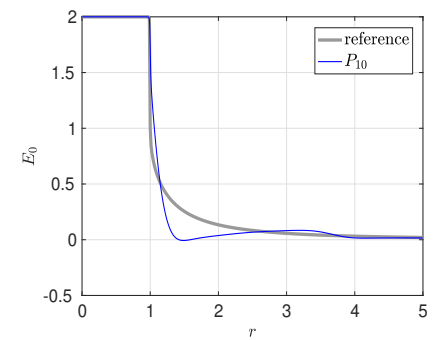

(a) Solution of $P_{10}$

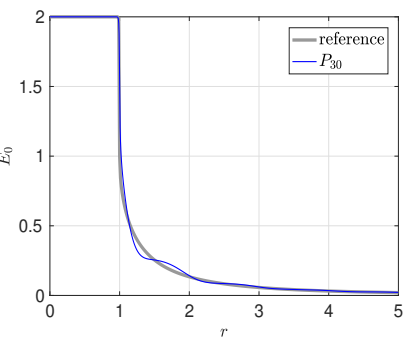

(b) Solution of $P_{30}$

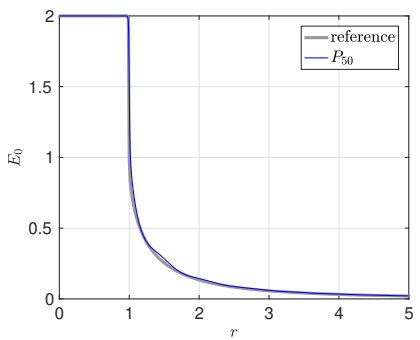

(c) Solution of $P_{50}$

Figure 8: Numerical results of the $P_{N}$ model for homogeneous sphere example 
results of $B_{2}$ model in Figure 7 all agree well with the analytical solution, showing that the $B_{2}$ model is valid to simulate both isotropic and anisotropic distribution functions.

Furthermore, notice that when $r<R$, because $\sigma=250 \gg 1$, the distribution function of the analytical solution is close to a constant. However, when $r \geq R$, the distribution function itself has a discontinuity with respect to $\mu$. Therefore, this problem not only has a discontinuity at the sphere with respect to spatial variable $r$, but also has a discontinuity with respect to velocity related variable $\mu$. Actually, it is this discontinuity which leads to the anisotropy of the distribution function. This phenomenon, which is often found in many practical applications, is a challenge for numerical simulations [1, 30]. However, the results of the $B_{2}$ model show that the $B_{2}$ model can handle the discontinuity well. On the other hand, according to the results of the $P_{N}$ model in Figure 8, the $P_{N}$ model can not handle this discontinuity, and we need a relative large order to obtain a satisfying result.

Example 4.4 (Static scattering atomsphere). This example is similar to test cases considered in [14, 1]. Consider the pure scattering steady-state radiative transfer equation

$$
\mu \frac{\partial I}{\partial r}+\frac{1-\mu^{2}}{r} \frac{\partial I}{\partial \mu}=-\sigma_{s} I+\frac{\sigma_{s}}{2} \int_{-1}^{1} I(\mu) \mathrm{d} \mu .
$$

where the computational region is $r \in[0, R], \sigma_{s}=r^{-n}$ with $n>1$. A vacuum boundary condition is prescribed at the right boundary. In addition, a point source is located at the origin, which emits L units of energy per unit time. This is the simplest spherical analogue of the classical Milne problem in the plane-parallel geometry.

The analytical result of this problem was studied in [14], where the researchers show the asymptotic behavior of $E_{0}$ when $r$ is sufficiently small and $r$ is sufficiently large. The tendency of $E_{0}$ can be formulated as

$$
E_{0} \propto\left\{\begin{array}{lr}
r^{-(n+1)}, & r \ll 1 ; \\
r^{-2}, & 1 \ll r \leq R .
\end{array}\right.
$$

We perform a set of simulations with $L=1, R=1$ and 100, and $n=\frac{3}{2}, 2$, and 3. When $R=1, N_{\text {cell }}=10000$ cells is used, and when $R=100$, we use $N_{\text {celll }}=100000$ cells. In Figure 9 we show the results of $E_{0}$ and the comparison with lines to determine the slopes. The numerical result for $E_{0}$ shown in Figure 9 is with the logarithm scale. Notice that $C, C_{1}$, and $C_{2}$ can be different in different subfigures. According to the results for different choices of $n$ in Figure 9, we know that the results of the $B_{2}$ model is consistent with the asymptotic analysis in (36). 


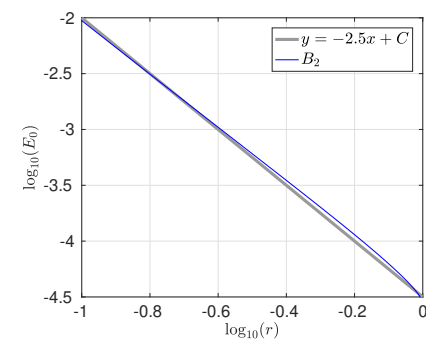

(a) solution of $E_{0}$ when $n=(b)$ solution of $E_{0}$ when $n=(c)$ $1.5, R=1$

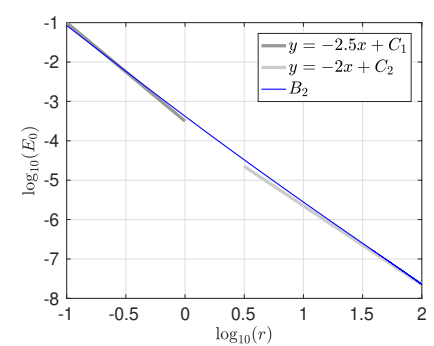

(d) solution of $E_{0}$ when $n=$ $1.5, R=100$

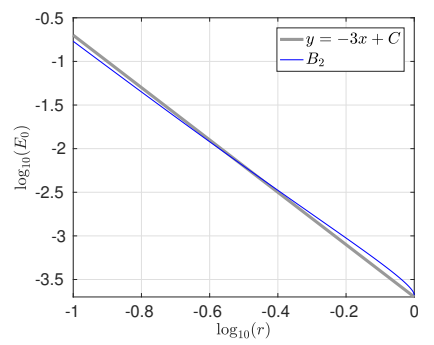

$2, R=1$

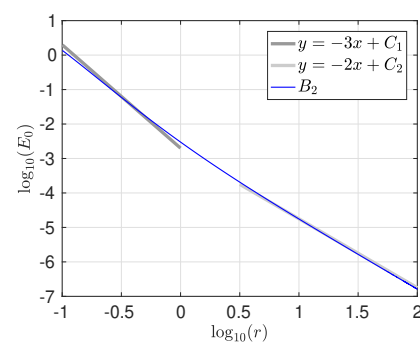

(e) solution of $E_{0}$ when $n=$ $2, R=100$

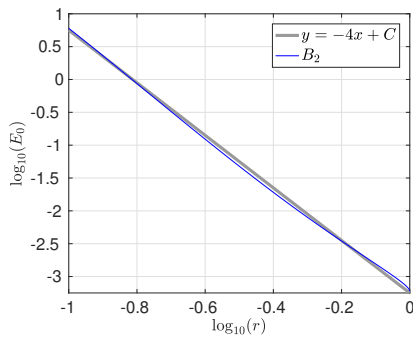

(c) solution of $E_{0}$ when $n=$ $3, R=1$

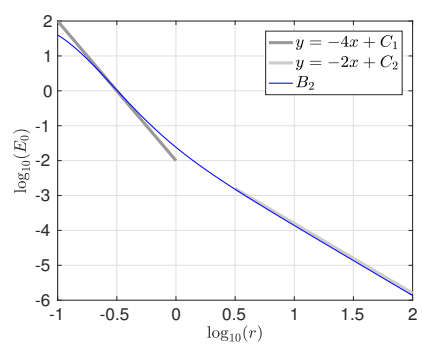

(f) solution of $E_{0}$ when $n=$ $3, R=100$

Figure 9: Numerical results for static scattering atomsphere.

Notice that in this example, $E_{0}$ tends to infinity when the radius $r$ tends to 0 . This means that in the geometry source term $\frac{1-\mu^{2}}{r} \frac{\partial I}{\partial \mu}$ is extremely stiff, which is a challenge in numerical simulations. However, the $B_{2}$ model yields correct result with a CFL number equals to 0.95 . Therefore, the $B_{2}$ model and the corresponding numerical scheme we adopt are valid and robust to simulate the RTE.

\section{Conclusion}

We extended the $B_{2}$ model for the radiative transfer equation in slab geometry, which was studied in [2], to the spherically symmetric case. The global hyperbolicity and realizability of the $B_{2}$ model can be verified, and its characteristic structure was analyzed in detail. One of the main advantages of the $B_{2}$ model is that its ansatz could capture highly anisotropic distribution functions, while at the same time it has explicit closing relationship. In our test cases where there are strongly peaked or discontinuous distribution functions the $B_{2}$ model shows better performance than the $P_{n}$ model, displaying its potential to approximate the RTE accurately and inexpensively. The next step is to extend the model to higher order. 


\section{Acknowledgements}

The work of R.L. and L.Z. is partially supported by Science Challenge Project, No. TZ2016002 and the National Natural Science Foundation of China (Grant No. 91630310 and 11421110001, 11421101). The work of W.L. is supported by NSFC Grant No. U1530401. The computational resources are supported by the high performance computing platform of Peking University, China.

\section{References}

[1] Ernazar Abdikamalov, Adam Burrows, Christian D. Ott, Frank Löffler, Evan O'Connor, Joshua C. Dolence, and Erik Schnetter. A new Monte Carlo method for time-dependent neutrino radiation transport. The Astrophysical Journal, 755(2):111, 2012.

[2] Graham W. Alldredge, Ruo Li, and Weiming Li. Approximating the $M_{2}$ method by the extended quadrature method of moments for radiative transfer in slab geometry. Kinetic \& Related Models, 9(2), 2016.

[3] L.S. Anusha, K.N. Nagendra, F. Paletou, and L. Léger. Preconditioned biconjugate gradient method for radiative transfer in spherical media. The Astrophysical Journal, 704(1):661, 2009.

[4] James E. Broadwell. Study of rarefied shear flow by the discrete velocity method. Journal of Fluid Mechanics, 19(03):401-414, 1964.

[5] T. A. Brunner and J. P. Holloway. Two-dimensional time dependent Riemann solvers for neutron transport. Journal of Computational Physics, 210:386399, 2005.

[6] Thomas A. Brunner and James Paul Holloway. One-dimensional Riemann solvers and the maximum entropy closure. Journal of Quantitative Spectroscopy and Radiative Transfer, 69(5):543-566, 2001.

[7] C.J. Cannon. Line transfer in two dimensions. The Astrophysical Journal, 161:255, 1970.

[8] Bruce T. Draine. Physics of the interstellar and intergalactic medium. Princeton University Press, 2010.

[9] Bruno Dubroca and J.L. Feugeas. Theoretical and numerical study on a moment closure hierarchy for the radiative transfer equation. Comptes Rendus de l'Academie des Sciences Series I Mathematics, 329(10):915-920, 1999. 
[10] Yuwei Fan, Jing An, and Lexing Ying. Fast algorithms for integral formulations of steady-state radiative transfer equation. to appear in Journal of Computational Physics, 2018.

[11] Yuwei Fan, Ruo Li, and Lingchao Zheng. A nonlinear moment model for radiative transfer equation in slab geometry. arXiv preprint arXiv:1812.11454, 2018.

[12] M. Gros, L. Crivellari, and E. Simonneau. An implicit integral method to solve selected radiative transfer problems. iv. the case of spherical geometry. The Astrophysical Journal, 489(1):331, 1997.

[13] Carole K. Hayakawa, Jerome Spanier, and Vasan Venugopalan. Coupled forward-adjoint Monte Carlo simulations of radiative transport for the study of optical probe design in heterogeneous tissues. SIAM Journal on Applied Mathematics, 68(1):253-270, 2007.

[14] D.G. Hummer and G.B. Rybicki. Radiative transfer in spherically symmetric systems. the conservative grey case. Monthly Notices of the Royal Astronomical Society, 152(1):1-19, 1971.

[15] Shi Jin and David Levermore. The discrete-ordinate method in diffusive regimes. Transport theory and statistical physics, 20(5-6):413-439, 1991.

[16] D.S. Kershaw. Flux limiting natures own way. Lawrence Livermore National Laboratory, UCRL-78378, 1976.

[17] Daniel Kitzmann, Jan Bolte, and A. Beate C. Patzer. Discontinuous Galerkin finite element methods for radiative transfer in spherical symmetry. Astronomy \& Astrophysics, 595:A90, 2016.

[18] Alexander D. Klose, Uwe Netz, Jürgen Beuthan, and Andreas H Hielscher. Optical tomography using the time-independent equation of radiative transferpart 1: forward model. Journal of Quantitative Spectroscopy and Radiative Transfer, 72(5):691-713, 2002.

[19] Paul Kunasz and Lawrence H. Auer. Short characteristic integration of radiative transfer problems: formal solution in two-dimensional slabs. Journal of Quantitative Spectroscopy and Radiative Transfer, 39(1):67-79, 1988.

[20] Edward W. Larsen and Jim E. Morel. Advances in discrete-ordinates methodology. In Nuclear Computational Science, pages 1-84. Springer, 2010. 
[21] Randall J. LeVeque. Finite volume methods for hyperbolic problems, volume 31. Cambridge university press, 2002.

[22] L.B. Lucy. Computing radiative equilibria with Monte Carlo techniques. Astronomy and Astrophysics, 344:282-288, 1999.

[23] Alexander Marshak and Anthony Davis. 3D radiative transfer in cloudy atmospheres. Springer Science \& Business Media, 2005.

[24] Ryan G. McClarren, James Paul Holloway, and Thomas A Brunner. On solutions to the $P_{n}$ equations for thermal radiative transfer. Journal of Computational Physics, 227(5):2864-2885, 2008.

[25] D. Mihalas. Stellar atmospheres, a series of books in astronomy and astrophysics, 1978.

[26] Dimitri Mihalas and Barbara Weibel Mihalas. Foundations of radiation hydrodynamics. Courier Corporation, 2013.

[27] Gerald N. Minerbo. Maximum entropy eddington factors. Journal of Quantitative Spectroscopy and Radiative Transfer, 20(6):541-545, 1978.

[28] G.C. Pomraning. The equations of radiation hydrodynamics. Pergamon Press, 1973.

[29] P.J. Schinder and S.A. Bludman. Radiative transport in spherical static spacetime - general relativistic tangent-ray method for the variable Eddington factors. 346:350-365, nov 1989.

[30] J.M. Smit, J. Cernohorsky, and C.P. Dullemond. Hyperbolicity and critical points in two-moment approximate radiative transfer. Astronomy and Astrophysics, v.325, p.203-211, 1997.

[31] Wenjun Sun, Song Jiang, and Kun Xu. An asymptotic preserving unified gas kinetic scheme for gray radiative transfer equations. Journal of Computational Physics, 285:265-279, 2015.

[32] Wasaburo Unno. Radiative transfer in spherical geometry. Publications of the Astronomical Society of Japan, 41:211-215, 1989. 